\title{
EXPRESSION OF COMPOSITE NUMBER AS PRIMES USING HYPER GRAPHS
}

\author{
${ }^{1}$ Meenakshi.K and ${ }^{2}$ Harisha.CS \\ ${ }^{1}$ Associate Professor, CMR Institute of Technology, Bangalore \\ meenakshik531@gmail.com \\ ${ }^{2}$ Assistant Professor, BTL Institute of Technology, Bangalore \\ harisha.cs@gmail.com
}

\begin{abstract}
The prime factorization theorem states that every integer greater than 1 can be expressed as a product of primes. Goldbach conjecture states that every even number greater than 2 can be expressed as a sum of 2 primes. In this paper we study the proof of prime factorization theorem using hypergraphs and we show that every sum of two or more primes gives a composite number.
\end{abstract}

\section{KEY WORDS}

prime factorization theorem, complete prime vertex composite edge weighted hypergraph

\section{INTRODUCTION}

Prime numbers have fascinated Mathematicians since the ancient Greeks. Euclid has given the first proof for existence of infinity of primes 2,3,5,7,11,13,.... Many Mathematicians were trying to find out the pattern for the same. Prime numbers have remained the main object of study for many years. Some of the important theorems or results related to prime numbers are the Fundamental theorem of Arithmetic, Fermat's theorem, Wilson's theorem, Perfect number conjectures, Prime Number theorem, Goldbach Conjecture and so on.

In number theory, the Fundamental theorem of Arithmetic also called as the Unique Factorization Theorem or the Unique Prime Factorization theorem states that every integer greater than 1 is either prime itself or can be expressed as the unique product of primes. The proof of the factorization is straight forward but the proof of its uniqueness is challenging. Factorisation of prime numbers is unique but factorization of composite numbers need not be unique. The fundamental theorem of arithmetic establishes the importance of prime numbers. Prime numbers are the basic building blocks of any positive integer in the sense that each positive integer can be constructed from the product of primes with one unique construction. Finding the prime factorisation of an integer allows derivation of all its divisors both prime and non prime. The property of uniqueness is powerful as the factorization can be thought of as a key for a number. The factorization technique is used to build powerful encryption techniques such as RSA. The prime factorization helps us to find the greatest common divisor and least common multiple among the numbers. The fundamental theorem ensures that additive and multiplicative arithmetic functions are completely determined by their values on the powers of prime numbers. Euclid has

DOI : $10.14810 /$ ijscmc.2015.4101 
discussed the proof of the same in two steps where in the first step every number is shown to be a product of zero or more primes and in the second step, the proof shows that any two representations may be unified into a single representation. Using Mathematical induction it was proved that there is a bag of primes corresponding to every positive integer. Christopher Walther and Stephen Schweitzer have discussed the machine supported proof of unique prime factorization theorem. They have demonstrated the use of verifun system with a verification of the theorem. They have illustrated the performance and operations of our system and presented the challenges encountered when working on this problem. Richard Hammack and Owen Puffenberger have used bipartite graphs for proving prime factor theorem. Its known that the class of connected non bipartite graphs with loops allowed obeys unique prime factorization over the direct product of graphs. The prime factorization is not unique in case of connected bipartite graphs. They have proved that prime factorization of a connected bipartite graph has exactly one bipartite factor.

Kalita has discussed the direct proof of Goldbach conjecture in 2000 and in 2006, has discussed the proof of the same in the opposite direction that is every sum of two primes gives all even numbers. Alex Vand etal showed that Goldbach conjecture is true for all the even numbers from 2 to $10^{18}$ Bichitra Kalita has discussed the graph theoretic proof of Goldbach conjecture. In his proof he has focussed on various properties of graphs relating to prime numbers. In this paper, we show that every sum or product of two or more primes gives composite number.

\section{DEFINITIONS}

\subsection{Graph}

A graph $G(V, E)$ consists of a finite non empty set $V$ called as set of vertices together with a set $E$ called the set of edges of undirected pair of distinct points of $\mathrm{V}$.

\subsection{Simple graph}

A graph $G(V, E)$ is said to be simple if it has no self loops and parallel edges.

\subsection{Multi graph}

A graph $G(V, E)$ is said to be a multi graph if it is not a simple graph.

\subsection{Degree of a vertex}

The number of edges incident with the vertex with self loops counted twice is called as the degree of that vertex. A vertex of a graph is called a pendent vertex if the degree of the vertex is one.

\subsection{Complete graph}

A graph $G(V, E)$ is said to be complete if there is an edge between every pair of vertices. A complete graph is also called as the universal graph or a clique. The degree of every vertex 
International Journal of Soft Computing, Mathematics and Control (IJSCMC),Vol. 4, No. 1, February 2015

in a complete graph is and it has 1 - nand it has $\frac{n(n-1)}{2}$ edges. The complete graph of vertices is denoted by $\mathrm{n}_{\mathrm{n}}$

\subsection{Bipartite graph}

A graph $G(V, E)$ is said to be bipartite graph if the vertex set $V$ is partitioned into two disjoint subsets say $V_{1}$ and $V_{2}$ consisting of $m$ and $n$ vertices whose edge has one vertex in $V_{1}$ and other vertex in $V_{2}$. It is said to be complete bipartite if every vertex of $V_{1}$ is incident with every other vertex of $V_{2}$. It is denoted by $K_{m, n}$. The bipartite graph $K_{1, n}$ is called as the star whose vertex set consist of 2 vertex sets $V_{1}$ and $V_{2}$ consisting of only one vertex and $n$ vertices respectively where one vertex of $V_{1}$ is incident with all the $n$ vertices of $V_{2}$.

\subsection{Weighted graph}

A weighted graph associates a label(weight) with every edge in the graph. Weights are usually real numbers. They may be restricted to rational numbers or integers.

\subsection{Hyper graph}

Hypergraphs, a generalisation of graphs have been widely and deeply studied in Berge $(1973,1984,1989)$ and quite often have proved to be a successful tool to represent and model concepts and structures in various areas of Computer Science and Discrete Mathematics. In Mathematics, a hypergraph is a generalisation of a graph in which an edge can connect any number of vertices. A hypergraph $H$ is a pair $H=(X, E)$ where $X$ is a set of elements called as nodes or vertices and $E$ is a set of non-empty subsets of $X$ called hyperedges or edges. Therefore $E$ is a subset of $\mathrm{P}(\mathrm{X})-\{0\}$ where $\mathrm{P}(\mathrm{X})$ is the power set of $X$. While graph edges are pairs of nodes, hyperedges are arbitrary sets of edges and can therefore contain an arbitrary number of nodes. Hypergraphs where all hyperedges have the same cardinality generally called as $k$ uniform hypergraph-- a hypergraph where all the hyperedges have size $\mathrm{k}$.

\subsection{B-arc}

A backward hyperarc or simply B-arc is a hyperarc $\mathrm{E}=(\mathrm{T}(\mathrm{E}), \mathbf{H}(\mathbf{E}))$ with $|H(E)|=\mathbf{1}$.

\subsection{F-arc}

A forward hyperarc or simply F-arc is a hyperarc $\mathrm{E}=(\mathrm{T}(\mathrm{E}), \mathrm{H}(\mathrm{E}))$ with $|T(E)|=1$

\subsection{A B-graph (or B-hypergraph)}

Is a hypergraph whose hyperarcs are B-arcs. A $\boldsymbol{F}$-graph (or F-hypergraph)is a hypergraph whose hyperarcs are F-arcs. A BF-graph (or BF-hypergraph) is a hypergraph whose hyperarcs are either B-arcs or F-arcs. 
International Journal of Soft Computing, Mathematics and Control (IJSCMC),Vol. 4, No. 1, February 2015

\section{NEW DEFINITIONS}

\subsection{Prime vertex hypergraph}

If the vertices of a hyper graph are prime numbers then it is called as the prime vertex hypergraph.

\subsection{Composite weighted hypergraph}

A weighted hypergraph $G(V, E)$ is said to be a composite weighted hypergraph if the weight of every edge is a composite integer.

\subsection{Complete hypergraph}

A hypergraph $G(V, E)$ is said to be complete if there is an edge between every pair of vertices.

\section{PROOF OF PRIME FACTORISATION THEOREM USING HYPER GRAPHS}

\subsection{Theorem}

All composite integers greater than or equal to 6 equals the product of primes can be obtained from union of complete hyper graph on $n$ primes and $\mathrm{BF}$ graphs for the same primes.

Proof Let $G(V, E)$ be the hypergraph on n vertices $(\mathrm{n} \geq 3)$ whose vertices are the consecutive primes say

$p_{1}, p_{2}, p_{3}, p_{4}, \ldots . p_{n} \ldots \ldots . .(n \geq 3)$ (primes pi $\left.\geq 2\right)$. Define the weight of every edge as the product of the

prime vertices. Find the minimum weight say w1 and the maximum weight say wi only from the

product of two vertices. Also find the integral part of half of the maximum weight that is $\left.\mid \frac{w_{i}}{2}\right\rceil$

Let this weight be $\mathrm{w}_{\mathrm{j}}$. We consider the composite numbers from $\mathrm{w}_{1}$ to $\mathrm{w}_{\mathrm{j}}$. Along with the given primes

$p_{1}, p_{2}, p_{3}, p_{4}, \ldots . p_{n} \ldots \ldots .(n \geq 3)$ we will have the new set of primes from $p_{1}$ to $p_{i}<\left\lceil\frac{w_{i}}{2}\right\rceil$

Step 1 Construction of the complete hyper graph

Given the prime vertices $p_{1}, p_{2}, p_{3}, p_{4}, \ldots, p_{n} \ldots \ldots(n \geq 3)$ where $p_{1}<p_{2} \quad<p_{3}<p_{4}<\ldots$

The hypergraph will have primes from $p_{1}$ upto less than $p_{i}=\left\lceil_{\mid}^{\lceil} \frac{w_{i}}{2}\right\rceil$. Using $p_{1, p_{2}^{*}, p_{1}^{*}, \ldots \ldots . . .}$ 
International Journal of Soft Computing, Mathematics and Control (IJSCMC),Vol. 4, No. 1, February 2015

As vertices and joining every vertex to every other vertex we get the complete hyper graph. The hyper edges consists of 2 edges, 3 edges,...... We get a special graph called as Prime Vertex Composite Edge Weighted Hypergraph (PVCEWHG). We express the composite numbers from $\mathrm{W}_{1}$ to $\mathrm{W}_{\mathrm{i}}$ as the product of primes. The composite numbers of the form $p_{i}{ }^{n}$ are the BF-hypergraphs of the form with the head or the tail node as 1 . Thus for any given set of primes, with the construction of Prime Vertex Composite Edge Weighted Hypergraph(PVCEWHG) and BF graphs we have product of primes giving composite numbers.

Example 1 Consider the set of primes 2,3,5

The minimum weight is 6 and the maximum weight is 15 . That is $w_{1}=6$ and $w i=15$. We define the new

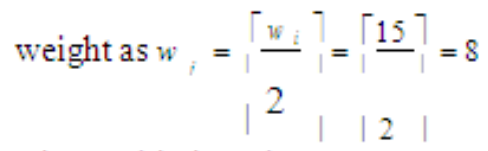

Along with the primes $2,3,5$ we consider the set of primes $<8$.

We construct the complete hyper graph using the prime vertices of the form $2^{\mathrm{n}}, 3^{\mathrm{n}}, 5^{\mathrm{n}}, 7^{\mathrm{n}}(\mathrm{n} \geq 0)$ and we take up edges comprising of 2 prime vertices and hyper edges comprising of 3 prime vertices, 4 vertices,....

The composite numbers from $\mathrm{w}_{1}=6$ to $\mathrm{w}_{\mathrm{i}}=15$ are $6,8,9,10,12,14,15$.

We find that product of primes giving all composite numbers from 6 to 15. ( Table 4.1)

Figure 4.1

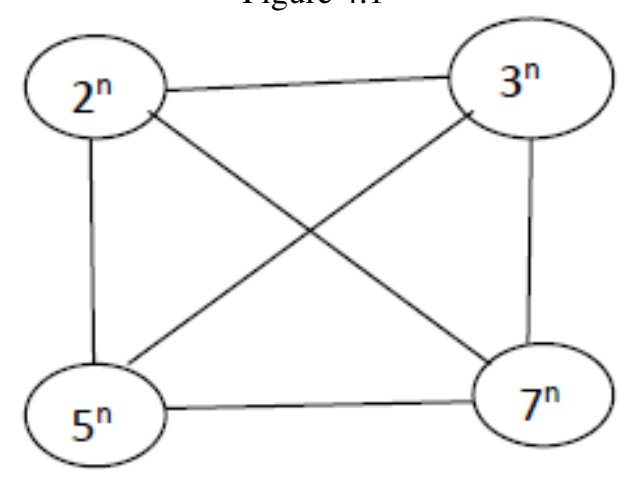

Example 2 Consider the set of primes 2,3,5,7

The minimum weight is 6 and the maximum weight is 35 . That is $\mathrm{w}_{1}=6$ and $\mathrm{w}_{\mathrm{i}}=35$.

We define the new weight as $w_{i}=\left\lceil\frac{w_{i}}{|2|}=\left\lceil\frac{35}{\mid 2}\right\rceil=18\right.$

Along with the primes 2,3,5 we consider the set of primes $<18$. We construct the complete 
International Journal of Soft Computing, Mathematics and Control (IJSCMC),Vol. 4, No. 1, February 2015 hyper graph using the prime vertices of the form $2^{\mathrm{n}}, 3^{\mathrm{n}}, 5^{\mathrm{n}}, 7^{\mathrm{n}}, 11^{\mathrm{n}}, 13^{\mathrm{n}}, 17^{\mathrm{n}}(\mathrm{n} \geq 0)$ and we take up edges comprising of 2 prime vertices comprising of 3 prime vertices,..... The composite numbers from $\mathrm{w}_{1}=6$ to $\quad \mathrm{Wi}_{\mathrm{i}}=35$ are $6,8,9,10,12,14,15,16,18,20,21,22,24,25,26,27,28,30,32,33,34,35$.

6

The following table shows that all composite numbers from 6 to 35 expressed as product of 2 or more primes.

Table 4.1

\begin{tabular}{|c|c|c|}
\hline $\begin{array}{c}\text { Composite } \\
\text { weighted edge }\end{array}$ & $\begin{array}{l}\text { e-(v1,v2) or } \\
\text { hyperedge } \\
\text { e-(v1,v2,v3) }\end{array}$ & $\begin{array}{l}\text { Expression of composite } \\
\text { number as product of primes }\end{array}$ \\
\hline 6 & $(2,3)$ & $6=2 \times 3$ \\
\hline 8 & $\left(2^{3}, 3^{0}\right)$ & $8=2^{3}$ \\
\hline 9 & $\left(3^{2}, 5^{0}\right)$ & $9=3^{2}$ \\
\hline 10 & $(2,5)$ & $10=2 \times 5$ \\
\hline 12 & $\left(2^{2}, 3\right)$ & $12=2^{2} \times 3$ \\
\hline 14 & $(2,7)$ & $14=2 \times 7$ \\
\hline 15 & $(3,5)$ & $15=3 \times 5$ \\
\hline 16 & $\left(2^{4}, 3^{0}\right)$ & $16=2^{4}$ \\
\hline 18 & $\left(2,3^{2}\right)$ & $18=2 \times 3^{2}$ \\
\hline 20 & $\left(2^{2} \times 5\right)$ & $20=2^{2} \times 5$ \\
\hline 21 & $(3,7)$ & $21=3 \times 7$ \\
\hline 22 & $(2,11)$ & $22=2 \times 11$ \\
\hline 24 & $\left(3,2^{3}\right)$ & $24=2^{3} \times 3$ \\
\hline 25 & $\left(5^{2}, 3^{0}\right)$ & $25=5^{2}$ \\
\hline 26 & $(2,13)$ & $26=2 \times 13$ \\
\hline 27 & $\left(3^{3}, 5^{0}\right)$ & $27=3^{3}$ \\
\hline 28 & $\left(7,2^{2}\right)$ & $28=2^{2} \times 7$ \\
\hline 30 & $(2,3,5)$ & $30=2 \times 3 \times 5$ \\
\hline 32 & $\left(2^{2}, 7^{0}\right)$ & $32=2^{2}$ \\
\hline 33 & $(3,11)$ & $33=3 \times 11$ \\
\hline 34 & $(2,17)$ & $34=2 \times 17$ \\
\hline 35 & $(5,7)$ & $35=5 \times 7$ \\
\hline
\end{tabular}

We have the constructed complete hyper graph below on seven vertices $\mathrm{v} 0, \mathrm{v} 1, \mathrm{v} 2, \mathrm{v} 3, \mathrm{v} 4, \mathrm{v} 5, \mathrm{v} 6$ corresponding to primes of the form $2^{\mathrm{n}}, 3^{\mathrm{n}}, 5^{\mathrm{n}}, 7^{\mathrm{n}}, 11^{\mathrm{n}}, 13^{\mathrm{n}}, 17^{\mathrm{n}}$ consisting of hyper edges . 
International Journal of Soft Computing, Mathematics and Control (IJSCMC),Vol. 4, No. 1, February 2015

Figure 4.2

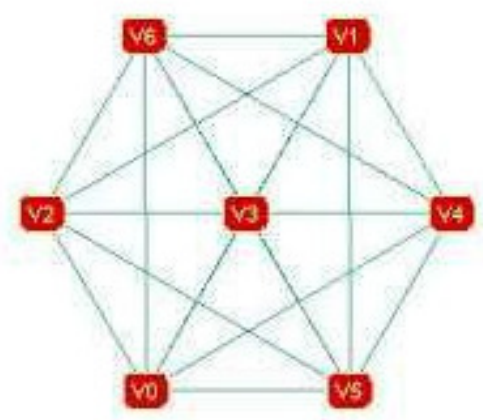

Example 3 Consider the set of primes 2,3,5,7,11

The minimum weight is 6 and the maximum weight is 77 . That is $\mathrm{w}_{1}=6$ and $\mathrm{wi}=77$.

$\left.\left.p i={ }_{\mid} \frac{w_{i}}{2}\right\rceil_{\mid}={ }_{\mid}{ }_{\mid} 77\right\rceil=39$. Along with the given primes we take up primes less than 39.

The hyper graph constructed will have 12 primes whose primes are $2,3,5,7,11,13,17,19,23,29,31,37$.

We construct the complete hyper graph with the prime vertices of the form $2^{\mathrm{n}}, 3^{\mathrm{n}}, 5^{\mathrm{n}}, 7^{\mathrm{n}}, 11^{\mathrm{n}}, 13^{\mathrm{n}}, 17^{\mathrm{n}}, 23^{\mathrm{n}}, 29^{\mathrm{n}}, 31^{\mathrm{n}}, 37^{\mathrm{n}}(\mathrm{n} \geq 0)$

We have product of primes giving all composite numbers from 6 to 77 shown in tables 4.1 and 4.2.

We have the constructed complete hyper graph on twelve vertices $\mathrm{v} 0, \mathrm{v} 1, \mathrm{v} 2, \mathrm{v} 3, \mathrm{v} 4, \mathrm{v} 5, \mathrm{v} 6, \mathrm{v} 7, \mathrm{v} 8, \mathrm{v} 9, \mathrm{v} 10, \mathrm{v} 11, \mathrm{v} 12$ which are primes of the form $\quad 2^{\mathrm{n}} 3^{\mathrm{n}} 5^{\mathrm{n}} 7^{\mathrm{n}} 11^{\mathrm{n}}, 1^{\mathrm{n}} 17^{\mathrm{n}}$, $23^{\mathrm{n}}, 29^{\mathrm{n}}, 31^{\mathrm{n}}, 37^{\mathrm{n}}(\mathrm{n} \geq 0)$ consisting of hyper edges.

Figure 4.3

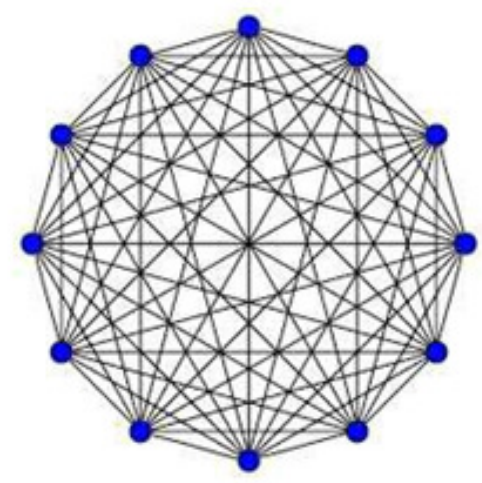


International Journal of Soft Computing, Mathematics and Control (IJSCMC),Vol. 4, No. 1, February 2015

Table 4.2

\begin{tabular}{|c|c|c|}
\hline Composite edge & e- $(\mathrm{V} 1, \mathrm{v} 2)$ or e-(v1,v2,V3) & $\begin{array}{l}\text { Expression of composite number as product } \\
\text { of primes }\end{array}$ \\
\hline 36 & $\left(2^{2}, 3^{2}\right)$ & $36=2^{2} \times 3^{2}$ \\
\hline 38 & $(2,19)$ & $38=2 \times 19$ \\
\hline 39 & $(3,13)$ & $39=3 \times 13$ \\
\hline 40 & $\left(5,2^{5}\right)$ & $40=5 \times 2^{3}$ \\
\hline 42 & $(2,3,7)$ & $42=2 \times 3 \times 7$ \\
\hline 44 & $\left(2^{2}, 11\right)$ & $44=2^{2} \times 11$ \\
\hline 45 & $\left(3^{2}, 5\right)$ & $45=3^{2} \times 5$ \\
\hline 46 & $(2,23)$ & $46=2 \times 23$ \\
\hline 48 & $\left(2^{4}, 3\right)$ & $48=2^{4} \times 3$ \\
\hline 49 & $\left(7^{2}, 3^{0}\right)$ & $49=7^{2}$ \\
\hline 50 & $\left(5^{2}, 2\right)$ & $50=5^{2} \times 2$ \\
\hline 51 & $(3,17)$ & $51=3 \times 17$ \\
\hline 52 & $\left(2^{2}, 13\right)$ & $52=2^{2} \times 13$ \\
\hline 54 & $\left(3^{5}, 2\right)$ & $54=3^{5} \times 2$ \\
\hline 55 & $(5,11)$ & $55=5 \times 11$ \\
\hline 56 & $\left(2^{3}, 7\right)$ & $56=2^{3} \times 7$ \\
\hline 57 & $(3,19)$ & $57=3 \times 19$ \\
\hline 58 & $(2,29)$ & $58=2 \times 29$ \\
\hline 60 & $\left(2^{2}, 3,5\right)$ & $60=2^{2} \times 3 \times 5$ \\
\hline 62 & $(2,31)$ & $62=2 \times 31$ \\
\hline 63 & $\left(3^{2}, 7\right)$ & $63=3^{2} \times 7$ \\
\hline 64 & $\left(2^{\circ}, 3^{0}\right)$ & $64=2^{0} \times 3^{\sigma}$ \\
\hline 65 & $(5,13)$ & $65=5 \times 13$ \\
\hline 66 & $(2,3,11)$ & $66=2 \times 3 \times 11$ \\
\hline 68 & $\left(2^{2}, 17\right)$ & $68=2^{2} \times 17$ \\
\hline 69 & $(3,23)$ & $69=3 \times 23$ \\
\hline 70 & $(2,5,7)$ & $70=2 \times 5 \times 7$ \\
\hline 72 & $\left(2^{3}, 3^{2}\right)$ & $72=2^{5 \times} 3^{2}$ \\
\hline 74 & $(2,37)$ & $74=2 \times 37$ \\
\hline 75 & $\left(5^{2}, 3\right)$ & $75=5^{2} \times 3$ \\
\hline 76 & $\left(2^{2}, 19\right)$ & $76=2^{2} \times 19$ \\
\hline 77 & $(7,11)$ & $77=7 \times 11$ \\
\hline
\end{tabular}

\section{EXPRESSION OF COMPOSITE NUMBER AS SUM OF PRIMES}

\subsection{Theorem}

All even weighted edges greater than or equal to 6 equals the union of weighted edges of complete prime edge-weighted hyper graph CPEWHG and the B-arc prime vertex hyper graph. 
International Journal of Soft Computing, Mathematics and Control (IJSCMC),Vol. 4, No. 1, February 2015

\section{Step 1 Construction of complete prime edge-weighted hyper graph CPEWHG}

Let $\mathrm{G}(\mathrm{V}, \mathrm{E})$ be the given graph. Attach primes $\mathrm{p}_{1}<\mathrm{p}_{2}<\mathrm{p}_{3}<\mathrm{p}_{4}<\mathrm{p}_{5}<\ldots$. (for the primes $\left.\mathrm{p}_{\mathrm{i}} \geq 2\right)$ to the vertices $v 1, v 2, v 3, \ldots . . n \geq 5$ for the primes(for the vertices $v_{i} \geq 5$ ). Define weight of the hyper edge as the sum of the primes. Join every vertex to every other vertex. The graph so obtained is called as complete prime edge-weighted hyper graph CPEWHG.

Figure 5.1

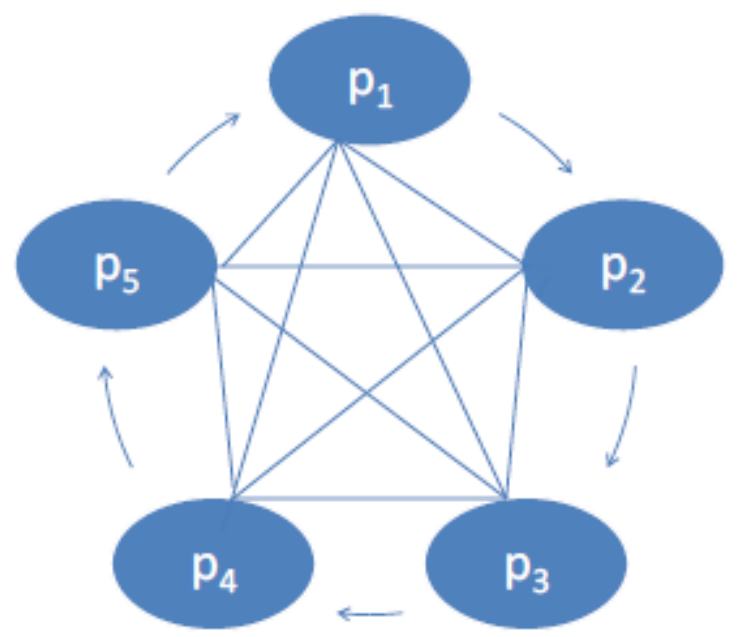

\section{Step 2 Construction of $B$ arcs}

For the given primes $\mathrm{p}_{1}<\mathrm{p}_{2}<\mathrm{p}_{3}<\mathrm{p}_{4}<\mathrm{p}_{5}<\ldots$. (for the primes $\mathrm{p}_{\mathrm{i}} \geq 2$ ) attached to the vertices $\mathrm{v} 1, \mathrm{v} 2, \mathrm{v} 3, \mathrm{v} 4, \mathrm{v} 5, \ldots \ldots$..(for the vertices $\mathrm{v}_{\mathrm{i}} \geq 5$ )

Construct the $B$ arcs whose vertices are primes of the form $p_{i}+p_{i}+p_{i}+\ldots \ldots$ The weight of the $\mathrm{B}$ arc is the number of arcs attached to the node.

Figure 5.2

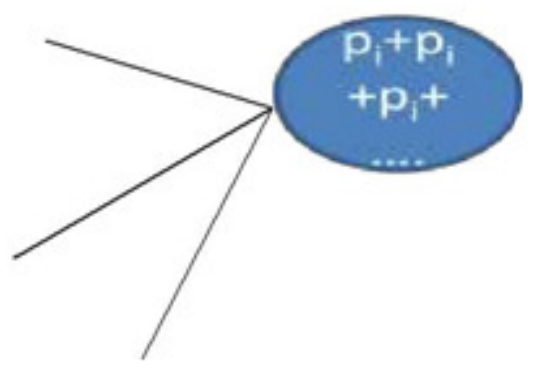

Using the above two constructions we can express every even number from 4 onwards as sum of primes. 
Example 1 Consider the given set of primes 2,3,5,7,11. The weight is defined as the sum of the consecutive primes. The minimum weight is 5 and the maximum weight is 28 . Construct the complete prime vertex edge weighted hyper graph.

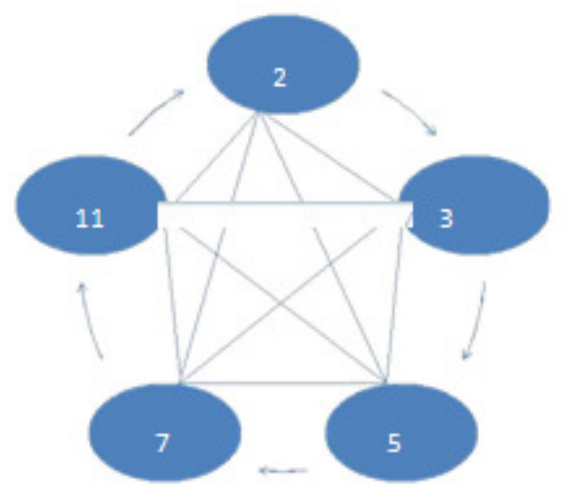

Step 2 For the given primes 2,3,5,7,11 construct the B arc as shown below . The vertices of the $\mathrm{B}$-arcs are of the form $\mathrm{p}_{\mathrm{i}}+\mathrm{p}_{\mathrm{i}}+\mathrm{p}_{\mathrm{i}}+\ldots \ldots \ldots$ the weight of the $\mathrm{B}$ arc is the total number of primes attached to the node.

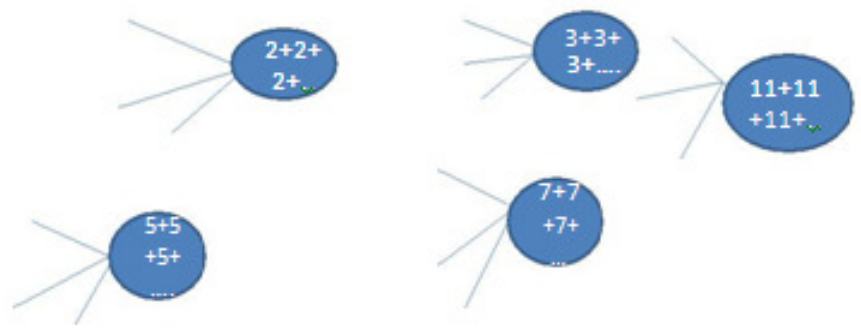

The union of complete prime vertex edge weighted hypergraph and the B-arc gives all even numbers as sum of primes.

Example 2 Consider the given set of primes 2,3,5,7,11,13. The minimum weight is 5 and the maximum weight is 41 . From the union of complete prime vertex edge weighted hyper graph and $\mathrm{B}$ arcs we have all even numbers from 4 to 40 as sum of primes shown in table 5.1. 
International Journal of Soft Computing, Mathematics and Control (IJSCMC),Vol. 4, No. 1, February 2015

Table 5.1

\begin{tabular}{|c|c|c|}
\hline Even number & $\begin{array}{c}\text { Edges comprising of } \\
\text { vertices }\end{array}$ & Sum of primes \\
\hline 4 & 2,2 & $2+2$ \\
\hline 6 & 3,3 & $3+3$ \\
\hline 8 & $2,2,2,2$ & $2+2+2+2$ \\
\hline 10 & $2,3,5$ or 5,5 & $5+3+2$ or $5+5$ \\
\hline 12 & $2,3,7$ & $3+3+3+3$ \\
\hline 14 & $2,5,7$ & $7+7$ \\
\hline 15 & $3,5,7$ & $5+5+5$ \\
\hline 16 & $2,3,11$ & $2+2+2+2+2+2+2+2$ \\
\hline 18 & $2,5,11$ & $3+3+3+3+3+3$ \\
\hline 20 & $2,7,11$ & $5+5+5+5$ \\
\hline 21 & $3,7,11$ & $7+7+7$ \\
\hline 22 & 11,11 & $11+11$ \\
\hline 24 & $3,3,3,3,3,3,3,3$ & $3+3+3+3+3+3+3+3$ \\
\hline 26 & $2,11,13$ & $11+13+2$ \\
\hline 28 & $7,7,7,7$ & $7+7+7+7$ \\
\hline 30 & $5,5,5,5,5,5$ & $5+5+5+5+5+5$ \\
\hline 32 & $2,2,2, \ldots$ & $2+2+2+\ldots 2(16$ times $)$ \\
\hline 34 & $2,2,2, \ldots$ & $2+2+2+\ldots 2(17$ times $)$ \\
\hline 36 & $5,7.11,13$ & $5+7+11+13$ \\
\hline 38 & $2,2,2, \ldots$ & $2+2+2+\ldots 2(19$ times $)$ \\
\hline 40 & $2,2,2, \ldots$ & $2+2+2+\ldots 2(20$ times $)$ \\
\hline
\end{tabular}

\section{CONCLUSION}

Using hyper graphs we have discussed the proof of the Fundamental Theorem of Arithmetic in the opposite direction and also we have expressed composite number as sum of primes.

\section{REFERENCES}

[1] Frank Harary, Graph Theory, Narosa Publishing House.

[2] B.Douglas, indtroduction to Graph Theory, Pearson Education (2002), 260-269.

[3] Bichitra Kalita, Graph and Goldbach Conjecture, International Journal of Pure and Applied Mathematics. Volume 82, No. 4 2013, 531-546.

[4] Paul Erdos Some applications of graph theory to number theory

[5] Noga Elon and P Erdos. Some applications of graph theory to additive number theory, European Journal of Combinatorics, 1985, 6, 201-203.

[6] Shariefuddin Pirzada and Ashay Dharwadker, Applications of Graph Theory, Journal of the Korean Scoeity for Industrial and Applied Mathematics, Vol.11, No.4, 2007. 
International Journal of Soft Computing, Mathematics and Control (IJSCMC),Vol. 4, No. 1, February 2015

\section{Authors}

\section{Dr.K.Meenakshi}

She is currently working as an Associate Professor in CMR Institute of Technology, Bangalore. She is having 17 years of teaching experience and has published papers in national and international journals.

\section{Mr.Harisha.CS.}

Harisha is currently working as a faculty in BTL Institute of Technology Bangalore. He is having 2 years of teaching experience and is doing research
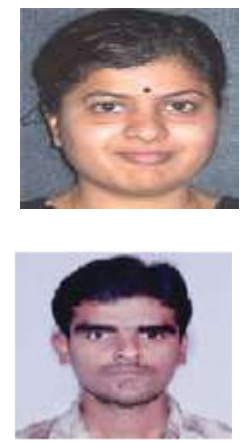